\title{
Enzymatic determination of Catechol oxidase and Protease from fruits (orange, apple) and vegetables (carrot, tomato)
}

\author{
Vanndita Bahl ${ }^{1}$, Parth Kapoor ${ }^{1}$, Prasidhi Tyagi ${ }^{2}$ andKameshwar Sharma YVR ${ }^{*}$ \\ ${ }^{1}$ (Biological Sciences, Sri Venkateswara College, University of Delhi, Delhi, India) \\ ${ }^{2}$ (Life Sciences, Sri Venkateswara College, University of Delhi, Delhi, India) \\ *(Department of Biochemistry, Sri Venkateswara College, University of Delhi, Delhi, India)
}

\begin{abstract}
The metabolic changes observed in any physical characters of fruits such as softness or colour change may be a result of changes in the activity of enzymes. The activity of plant polyphenoloxidases can cause enzymatic browning of fruits and vegetables and render food unfit for consumption due to formation of benzoquinone. Proteases degrade polypeptides and facilitate seed germination, leaf senescence, storage and release of amino acids for transport to actively growing regions. Kinetic activity is significant in understanding the process of fruit and vegetable ripening. It is evident from the literature that these enzymes (Catechol oxidase and Protease) have a prominent role in the immunity and defence mechanism.

The present study has been focused on the enzymatic profiles of Catechol oxidase and Protease in fruits (Orange and Apple) and vegetables (Tomato and Carrot) which can be helpful in isolating and characterizing the enzymes from the above sources. Catechol oxidase and Protease were assayed using catechol and egg albumin as substrates, respectively. The Catechol oxidase activity was determined by measuring the change in colour of solution comprising of substrate and fruit/ vegetable extract due to formation of chromophoric substance, benzoquinone. Protease was assayed by measuring the rate at which the solution of egg albumin got digested by the activity of enzyme present in fruit/vegetable juice.

An increase in the absorbance due to formation of benzoquinone was observed in tomato, orange and apple juices due to increased activity on enzyme Catechol oxidase implying to more ripening of fruits/ vegetables as compared to carrot juice where the absorbance value was found to decrease indicating less enzyme activity. Protease enzyme activity in tomato juice was found to decrease due to increase in absorbance as lesser amount of egg albumin was digested by the enzyme. However Protease activity was found to increase in orange juice sample. From these results it is evident that, protease has a significant and prominent role in ripening and softening of the fruit (orange) as compared to vegetable (tomato).
\end{abstract}

Keywords - apple, benzoquinone, catechol oxidase, carrot, orange, protease, tomato

\section{INTRODUCTION}

The metabolic changes that occur in ripening of fruits and vegetables reflect changes in the activity of enzymes within their tissues. Changes observed in any of the physical characters of the fruit, such as softness or color change, may be the result of changes in the activity of one or more of the enzymes. An enzyme may be responsible for degrading a particular component of the plant cell wall, or degradation of a particular pigment leading to a change in color. Some of these naturally occurring enzymes, such as pectinase and cellulase, are used in the food processing industry [1].

\subsection{Polyphenol oxidase (Catechol oxidases)}

Polyphenol oxidases are a widespread group of enzymes found in plants, fungi, bacteria and animals. In plants, these enzymes are usually found in the chloroplasts, although they can be released from here during ripening or senescence. Polyphenol oxidase (PPO) enzyme is able to catalyze the transformation of an array of aromatic compounds that have two adjacent phenolic groups on them. This includes a number of polyphenols in plants that act as antioxidants. These copper-containing enzymes oxidize the phenolic groups to reactive oxygen molecules known as quinones, which continue reacting with each other and other cellular factors to form brown spots known as melanin. These melanins form barriers and have antimicrobial properties which prevent the spread of infection in plant tissues. Plants, which exhibit comparably high resistance to climatic stress, have been shown to possess relatively higher polyphenol oxidase levels than susceptible varieties. Browning causes the deterioration of fruits and vegetables, resulting in large economic losses. Polyphenol oxidase also occurs in animals and is thought to increase disease resistance in insects and crustaceans. There are two types of PPOs. viz., Catechol oxidase (catecholase) can catalyze aromatic groups with two adjacent phenols, a diphenol, to become two quinones. The other is tyrosinase (monophenolase) activity, which involves introducing one atom of oxygen onto a carbon atom of an aromatic group with one phenolic group on it, changing it to a diphenol, with the two phenolic groups being adjacent to each other. Then, the enzyme will mediate catechol oxidase 
activity on the molecule, transforming it to a molecule with two quinones. Tyrosinase enzymes are also responsible for producing the melanin pigments in our skin. These quinones frequently continue reacting with each other and nearby proteins to generate brown pigments. Such pigments result in the enzymatic browning of fruits and vegetables and often render food unfit for consumption [2].

In the reaction Catechol is oxidised initially to the orange coloured compound benzoquinone which is then converted to melanins. The conversion to melanin is spontaneous, but slow. Food processing and cooking often involve procedures which are intended to inhibit the action of polyphenoloxidases.

\subsection{Proteases}

Digestion is the process which helps an organism to convert food molecules into forms that are nutritionally useful to that organism. In the case of animals possessing an alimentary canal, numerous extracellular enzymes are secreted into the lumen of this structure so that ingested food items may be transformed into forms that can be absorbed through the epithelium [3]. It has been established that fishes and higher vertebrates utilize the same enzymes and hormones in the breakdown of proteins into amino acids [4]. In fish, the specific levels of digestive enzyme activities are dependent on age, diet, season, and/or ambient temperature [5]. Proteases attack peptide bonds of proteins and polypeptides, thereby converting large peptide chains into shorter polypeptide segments. There are two basic types of proteases, the endopeptidases and the exopeptidases. In case of former, the enzyme attacks bonds located deep within the substrate protein, thus transforming large peptide chains into shorter peptide segments. Exopeptidases function by converting these shorter segments, located near the end of a peptide chain, into free amino acids, dipeptides and tripeptides. Trypsin, chymotrypsin and elastase are endopeptidases occurring in the alimentary canal of perhaps virtually all invertebrates and vertebrates possessing this digestive structure [6]. The degradation of proteins is important for plant development. Proteins may become unwanted because they have become damaged, or the requirement of the cell has changed making previously synthesized proteins redundant. In several key stages of plant development such as during germination of seeds and during leaf senescence, storage and other proteins are degraded to release amino acids for transport to the most actively growing regions of the plants. Protease activity is low in the youngest leaves and very high in old senescing leaves [7].

Over one third of known proteolytic enzymes are serine proteases. This large family of enzymes, hydrolyzes the peptide bond of substrate via a nucleophilic serine residue in the active site,

Ser-195 (chymotrypsin numbering), arranged with Asp-102 and His-57 into a catalytic triad [8].Rawlings et al have devised a classification scheme based on statistically significant similarities in sequence and structure of all known proteolytic enzymes and terms this database MEROPS. This classification system divides proteases into clans based on catalytic mechanism and families on the basis of common ancestry [9].

At present, over 48,000 peptidase gene sequences have been classified into 50 clans and 184 families. Over 18,000 serine proteases are grouped into 12 clans and 40 families. X-ray crystal structures have been determined for a few representatives of each family. Clan PA peptidases bearing the chymotrypsin-fold are the largest and best studied family of serine proteases. They are abundantly represented in vertebrates, but rare constituents of plant genomes. Prototypic members of this clan are trypsin and chymotrypsin, which cleave polypeptide chains on the C-terminal side of a positively charged side-chain (Arg or Lys) and large hydrophobic residue (Phe, Trp, Tyr), respectively. Initiation of protease activity is largely controlled by zymogen activation. Cessation of protease activity in vivo is achieved by one of three classes of protease inhibitors - canonical, noncanonical and serpin [10].

In many members of the family, activity and specificity are allosterically regulated by macromolecular ligands, or small cations like $\mathrm{Na}^{+}$and $\mathrm{Ca}^{2+}[11]$.The three serine proteases of the chymotrypsin-like clan that have been studied in greatest detail are chymotrypsin, trypsin, and elastase. All three enzymes are synthesized by the pancreaticacinar cells, secreted in the small intestine and are responsible for catalyzing the hydrolysis of peptide bonds. All three of these enzymes are similar in structure from their X-ray structures. The differing aspect lies in the peptide bond that is being cleaved; this is called the scissile bond. The different enzymes, like most enzymes, are highly specific in the reactions they catalyze. Each of these digestive serine proteases target different regions of a polypeptide chain based upon the side chains of the amino acid residues surrounding the site of cleavage. Chymotrypsin is responsible for cleaving peptide bonds following a bulky hydrophobic amino acid residue. Preferred residues include phenylalanine, tryptophan, and tyrosine, which fit into a snug hydrophobic pocket. Trypsin is responsible for cleaving peptide bonds following a positively-charged amino acid residue. Instead of having the hydrophobic pocket of the chymotrypsin, there exists an aspartic acid residue at the base of the pocket. This can then interact with positively-charged residues such as arginine and lysine on the substrate peptide to be cleaved. Elastase is responsible for cleaving peptide bonds following a small neutral amino acid residue, such as alanine, glycine and valine. The pocket that is in "trypsin" and "chymotrypsin" is now partially filled with valine and threonine, rendering it into a mere depression, which can accommodate 
these smaller amino acid residues. The combination of these three enzymes makes an incredibly effective digestive team and is primarily responsible for the digestion of proteins.

\subsection{Catalytic Mechanism of Serine Proteases}

The main player in the catalytic mechanism in the chymotrypsin and subtilisin clan enzymes mentioned earlier is the catalytic triad. The triad is located in the active site of the enzyme, where catalysis occurs and is preserved in all serine protease enzymes. The triad is a coordinated structure consisting of three essential amino acids: histidine(His 57), serine (Ser 195) (hence the name "serine protease") and aspartic acid (Asp 102). Located very close to one another near the heart of the enzyme, these three key amino acids play an essential role in the cleaving ability of the proteases. In the event of catalysis, an ordered mechanism occurs in which several intermediates are generated. The catalysis of the peptide cleavage can be seen as a ping-pong catalysis, in which a substrate binds (in this case, the polypeptide being cleaved), a product is released (the N-terminus "half" of the peptide), another substrate binds (in this case, water), and another product is released (the C-terminus "half" of the peptide). Each amino acid in the triad performs a specific task. The serine has an - $\mathrm{OH}$ group that is able to act as a nucleophile, attacking the carbonyl carbon of the scissile peptide bond of the substrate. A pair of electrons on the histidine nitrogen has the ability to accept the hydrogen from the serine - $\mathrm{OH}$ group, thus coordinating the attack of the peptide bond. The carboxyl group on the aspartic acid in turn forms hydrogen bonds with histidine, making the pair of electrons much more electronegative. The whole reaction is summarized as follows (Fig.2).

- The polypeptide substrate binds to the surface of the serine protease (enzyme) in such a way that scissile bond is inserted into the active site of the enzyme, with the carbonyl carbon of this bond positioned near the nucleophilic serine.

- The serine -OH attacks the carbonyl carbon, and the nitrogen of the histidine accepts the hydrogen from the $-\mathrm{OH}$ of the [serine] and a pair of electrons from the double bond of the carbonyl oxygen move to the oxygen. As a result, a tetrahedral intermediate is generated.

- The bond joining the nitrogen and the carbon in the peptide bond is now broken. The covalent electrons creating this bond move to attack the hydrogen of the histidine, breaking the connection. The electrons that previously moved from the carbonyl oxygen double bond move back from the negative oxygen to recreate the bond, generating an acyl-enzyme intermediate.

- Now, water comes into the reaction. Water replaces the N-terminus of the cleaved peptide, and attacks the carbonyl carbon. Once again, the electrons from the double bond move to the oxygen making it negative, as the bond between the oxygen of the water and the carbon is formed. This is coordinated by the nitrogen of the histidine which accepts a proton from the water. Overall, this generates another tetrahedral intermediate.

- In a final reaction, the bond formed in the first step between the serine and the carbonyl carbon moves to attack the hydrogen that the histidine has just acquired. Then electron-deficient carbonyl carbon re-forms the double bond with the oxygen. As a result, the C-terminus of the peptide is now ejected. It was discovered that additional amino acids of the protease, Gly 193 and Ser195, are involved in creating an oxyanion hole. Both Gly193 and Ser195 can donate backbone hydrogens for hydrogen bonding.

- When the tetrahedral intermediate of step 1 and step 3 are generated, the negative oxygen ion, having accepted the electrons from the carbonyl double bond fit perfectly into the oxyanion hole. In effect, serine proteases preferentially bind to the transition state and the overall structure is favoured, lowering the activation energy of the reaction. This "preferential binding" is responsible for much of the catalytic efficiency of the enzyme.

The activity of catechol oxidase and protease were done in the fruits (orange and apple) and vegetables ( tomato and carrot).

\subsection{Apple}

The apple is the pomaceous fruit of the apple tree, species Malusdomestica in the rose family Rosaceae. It is one of the most widely cultivated fruit trees, and the most widely known of the many members of genus Malus that are used by humans. It is a health vitalizer, improves intelligence, cleans the digestive tract, stops excessive bleeding, and regulates menstruation. It is rich in phosphates and vitamins and is good for hair, skin, nails and eyes. It has antioxidant compounds and the apple fibers lower blood cholesterol levels and raises HDL. The vitamins which are found in apple juice include nicotinic acid, folic acid, riboflavin, vitamin C, vitamin A, carotene, B6, thiamine, biotin [12].

\subsection{Orange}

The main nutritional content in oranges is ascorbic acid. This powerful antioxidant neutralizes harmful elements inside the body. Vitamin $\mathrm{C}$ also energizes the absorption of non heme iron and therefore reduces iron deficiency. As a whole the ascorbic acid content in orange keeps the immune system strong and healthy. Ascorbic acid is essential for both expectant mother and baby because it is the cementing agent that holds new 
cells together. It helps babies grow and make strong bones and teeth. It also helps the expectant mother's body to absorb more iron.

Orange juice is a natural source of potassium and sodium and is cholesterol free. Evidence also shows that nutrients present in orange juice, for example potassium, folate and ascorbic acid, may shield against coronary diseases. One study indicated that drinking three 8-ounce portions of orange juice each day increased HDL (Good) cholesterol by $21 \%$ and reduced LDL (Bad) cholesterol by $16 \%$. Additionally, the serum amounts of vitamin $\mathrm{C}$ and folate also increased. Orange juice comes with vitamins that can help some cancers.

\subsection{Tomato}

Raw tomato juice is a good source of lycopene, which is known for its cancer preventing properties. In addition, it stimulates blood circulation and helps lower blood pressure. Lycopene is fat soluble, so adding a few drops of oil is recommended for better absorption. Raw tomato juice contains potassium, an essential mineral macronutrient in human nutrition. Low potassium in the body fluids leads to general weakness and malaise. Homemade tomato juice is rich in vitamin $\mathrm{C}$, which is known for its immune enhancing properties. Raw tomato juice improves the appetite and digestion. Drinking tomato juice mixed with spinach juice will activate liver and help relieve and prevent constipation. Being rich in vitamin $\mathrm{C}$ and potassium, raw tomato juice is also good for pregnant woman to supply nutrition for the body [13].

\subsection{Carrot}

Carrot contains the maximum amount of beta-carotene. In our body, beta-carotene is converted into Vitamin A, which assists in improving our eyesight and the functioning of our immune system, strengthening our bones and teeth, preventing possible problems with the functioning of the thyroid gland and has a positive effect on our hair, nails and skin. Vitamin A also has cleansing effect, cleans liver from fat and other unwanted elements. The list of microelements and nutrients which are contained in carrot includes vitamins C, B, E, D \& $\mathrm{K}$, proteins, potassium, calcium, phosphorus, zinc, aluminium, sodium, manganese, iron, copper and other minerals. Carrot contains more amount of nicotinic acid, which is important for metabolism of fats and lipids. The vegetable is a great source of natural magnesium, which assists in decreasing bad cholesterol levels in the body, helps relieve spasms and strengthen our blood vessels. Carrot juice has very good anti inflammatory, anti bacterial, antiseptic, anti cancerous, anti ageing and antioxidant properties, which can prevent degenerative processes in the cells. Carrot juice is helpful in lowering the symptoms of stress and normalizing functions of the digestive system [14].

Review of literature shows that these enzymes (Catechol oxidase and Protease) have a prominent role in the immunity and defence mechanism. The present study has been focused on the enzymatic profiles of Catechol oxidase and Protease in fruits (orange and apple) and vegetables (tomato and carrot). Enzymatic profile is a pilot study helpful in isolating and characterizing the enzymes from the above sources.

\section{Materials And Methods}

Plant sources like apple,orange, tomato, andcarrot were purchased from local market. Chemicals like pH-7 buffer, $0.1 \%$ catechol, were of analytical grade. Colorimeter (1312, Systronics, Mumbai, India), mixer/grinder, centrifuge (Superspin R-V/Fm, Plasto crafts, New Delhi, India), water bath, Muslin cloth, eggs, wire gauze, tripod stand, burner, were used for the experimental work. Glassware, funnel, pipettes, beakers, test tubes (Borosil, India).

\subsection{Assay of Catechol oxidase}

Assay is based on the change from a colourless solution of catechol to coloured benzoquinone and is measured by using a colorimeter. A fruit extract is added to a solution of catechol and the rate of formation of coloured benzoquinone is measured [2]. The faster the rate of increase in absorbance of the reaction mixture, the greater is the polyphenol oxidase activity of the fruit extract.

Extract of fruit or vegetable was prepared by grinding them in a mortar or blending with an equivalent mass of water in the ratio of $1: 1$. Strained the extract through muslin cloth and centrifuged (RPM-3000, time-15 minutes, temperature $-15^{\circ} \mathrm{C}$ ) the filtrate to remove the remaining solids. Separated the supernatant for further study. Assay was performed by taking $2 \mathrm{ml}$ of standard $\mathrm{pH} 7.0$ and $2 \mathrm{ml}$ of $0.1 \%$ catechol. Eventually time was noted and $0.1 \mathrm{ml}$ enzyme extract was added with immediate mixing of the tubes. Absorbance (A $540 \mathrm{~nm}$ for tomato, orange and apple extracts, A $480 \mathrm{~nm}$ for carrot extract) was measured at every 20 seconds using colorimeter.

\subsection{Protease assay}

Protease enzymes are added to a milky colloidal suspension of egg albumen. As the protease enzymes digest the suspended particles of proteins, the mixture becomes more transparent. The absorbance changes in the reaction mixture are taken by using a colorimeter. The protease content can be assayed by measuring the rate at which the solution of egg albumen and extract becomes clearer [2]. 


\subsection{Preparation of the egg albumen colloidal suspension (enzyme substrate)}

Separated the white of a single egg into a beaker $(250 \mathrm{ml})$ and $150 \mathrm{ml}$ of distilled water was added with constant stirring of the mixture. The mixture became quite cloudy as a result of the denaturation of some of the egg albumen by the water. Placed the beaker on a tripod stand and heated with a Bunsen burner, until the mixture boiled, stirring it regularly. Mixture was allowed to cool and then it was decanted through two or three layers of muslin cloth into another beaker. This created a homogenous milky colloidal solution.

In a test tube, $2 \mathrm{ml}$ of $\mathrm{pH} 7.0$ buffer was added followed by $2 \mathrm{ml}$ of albumen substrate solution and 1 $\mathrm{ml}$ of fruit extract. The contents were mixed and the reaction mixture was placed in the colorimeter. Reagent blank was maintained for this reaction and absorbance was taken. Placed the reaction mixture(s) in a water bath at $30^{\circ} \mathrm{C}$ to promote the activity of any protease enzymes present. Absorbance (A $540 \mathrm{~nm}$ ) was taken at regular time intervals, until no further change in absorbance was detected.

\section{FIGURES AND TABLES}

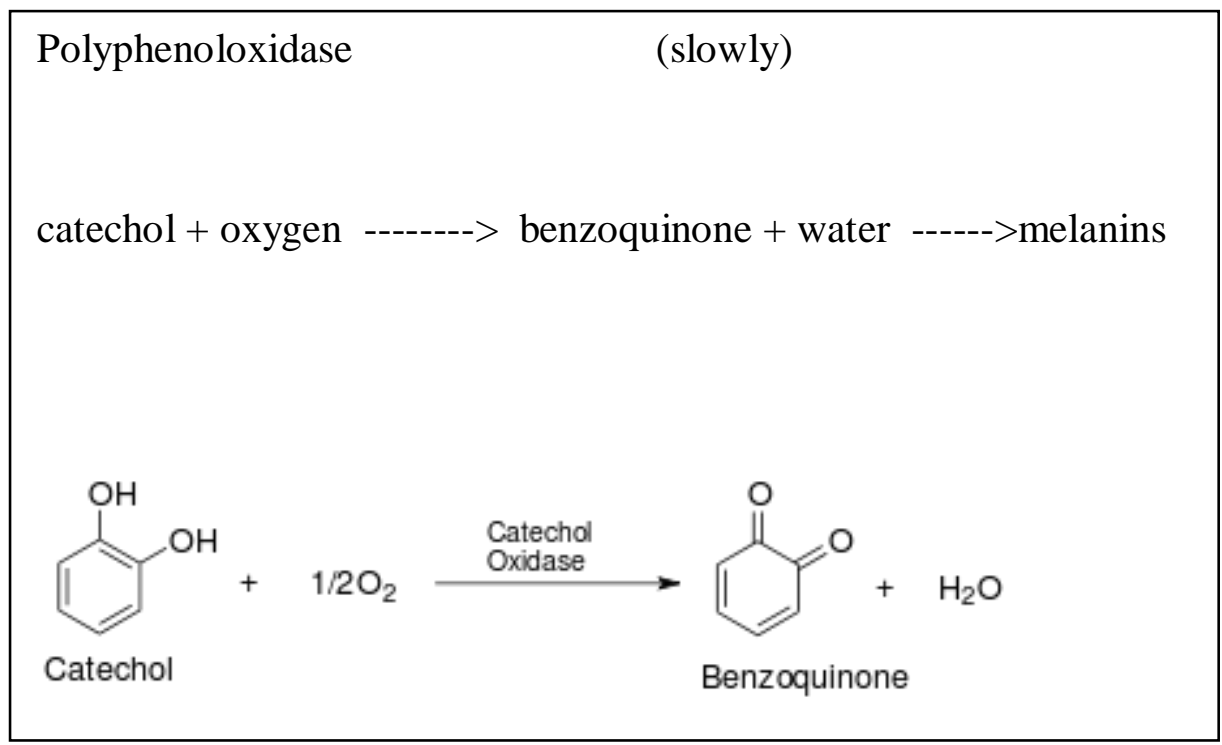

Fig.1. Reaction Mechanism of Catechol oxidase

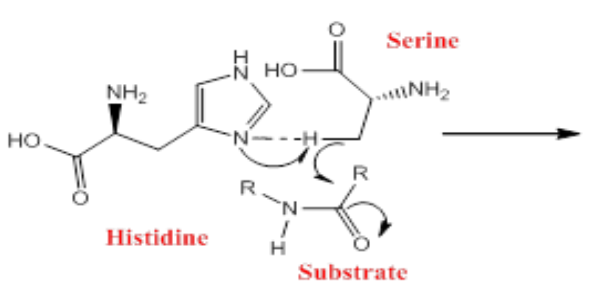

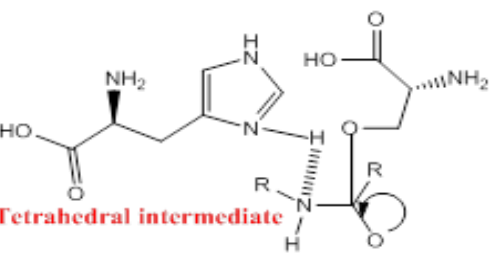

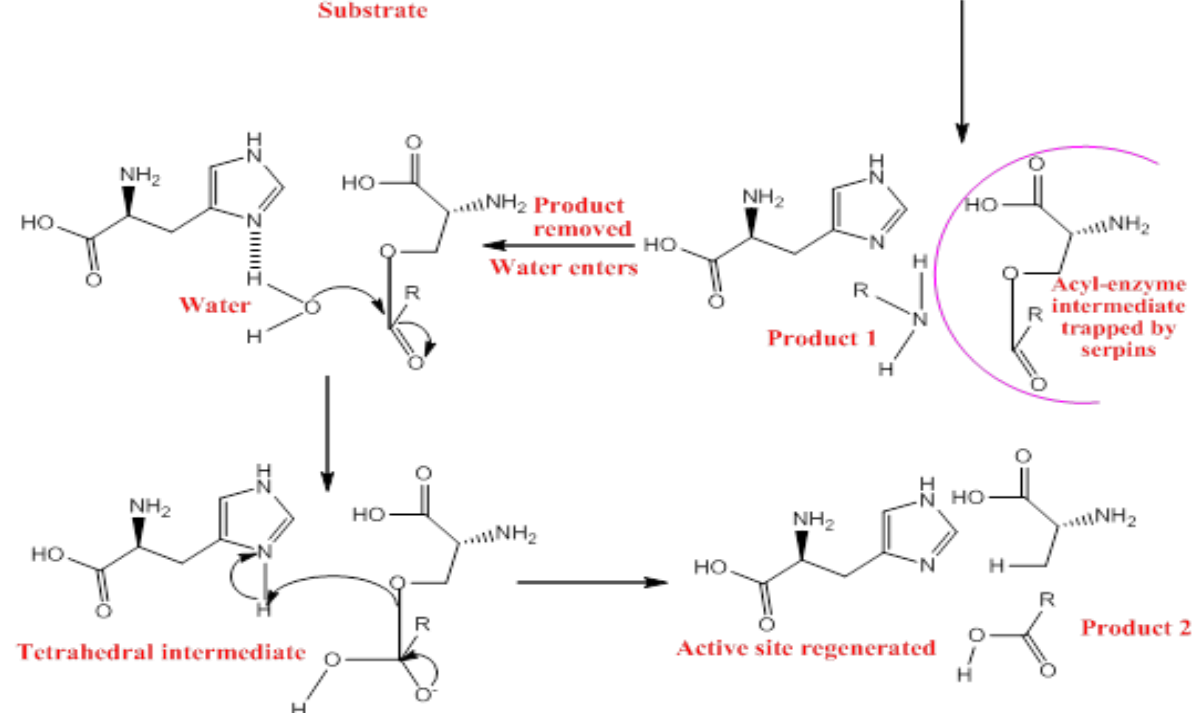

Fig.2. Reaction Mechanism of Serine Protease 


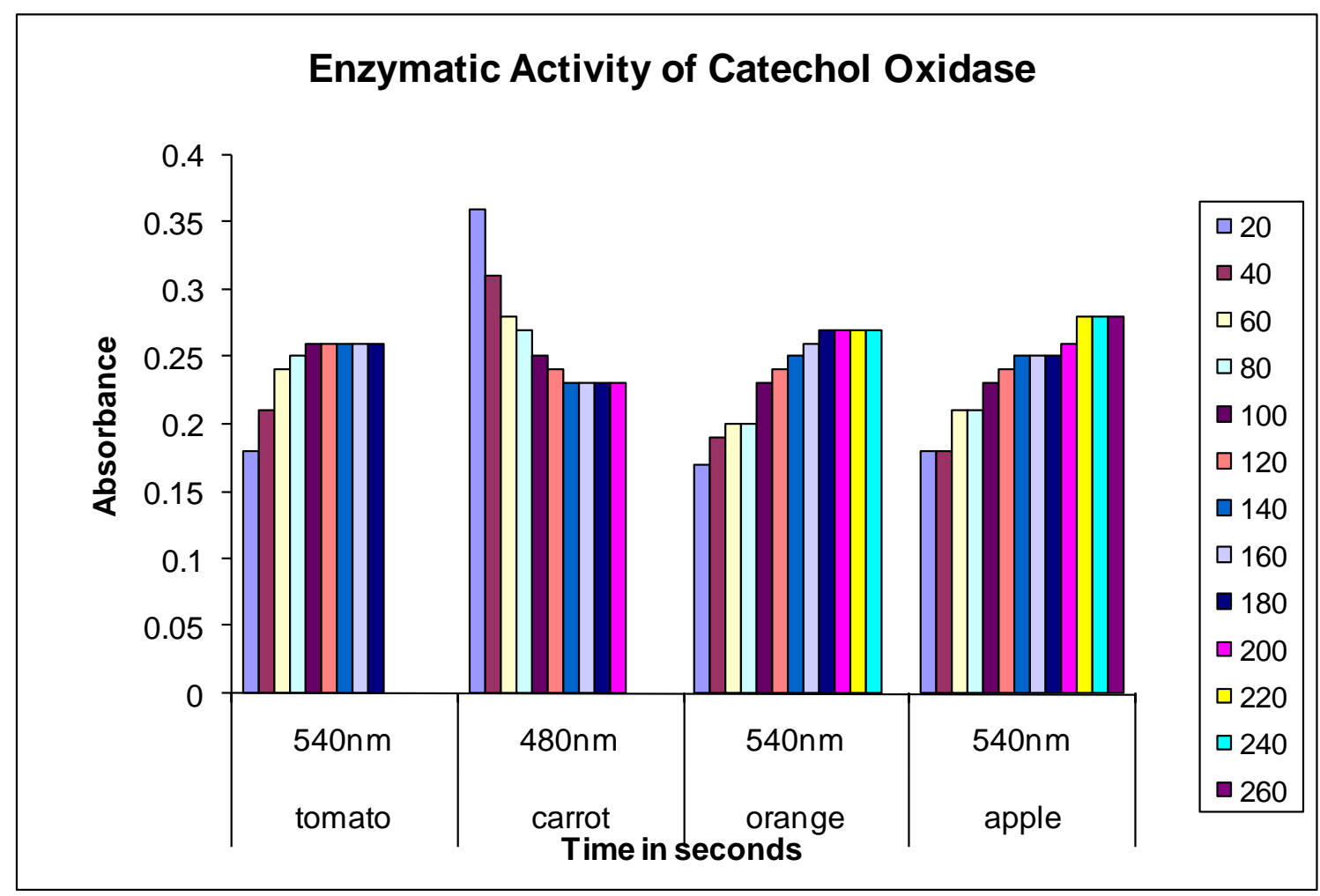

Fig.3. Enzyme activity of Catechol Oxidase of Tomato, Carrot, Orange and Apple with respect to time intervals in seconds.

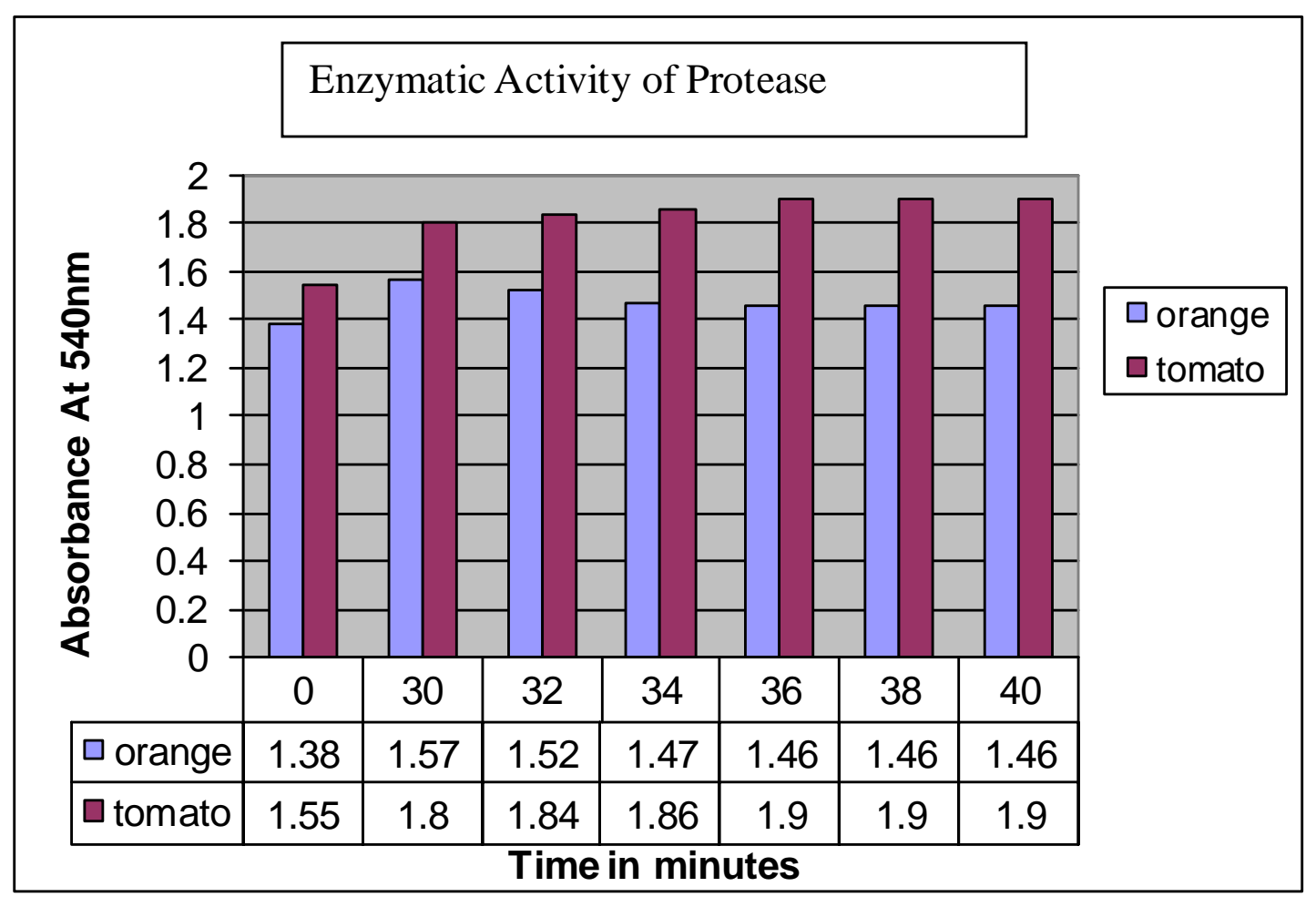

Fig.4. Protease activity of Orange and Tomato juice extract.Absorbance at $540 \mathrm{~nm}$ vs. time in minutes. 


\subsection{Catechol oxidase}

\section{RESULTS AND DISCUSSION}

Absorbance value of tomato extract for Catechol oxidase was found to increase up to 100 seconds and then became constant thereafter. The Catechol oxidase activity of carrot was found to decrease initially and became constant after $1 \mathrm{~min} 40$ seconds (Fig.3). This shows that the activity of carrot decreased with respect to tomato. The activity of Polyphenol oxidase leads to the formation of benzoquinone and hence the absorbance increased in tomato whereas in carrot the benzoquinone formation was very slow or negligible.

Activity of orange juice extract was found to increase for 60 seconds initially and then became constant. The absorbance was then found to increase and finally became constant after 3 minutes. The absorbance value of apple juice extract was found to be constant for up to 40 seconds and was then found to increase and finally after 4 minutes it become constant. (Variations of activity in between may be because of improper mixing of enzyme with the substrate). The enzymatic activity though was found to increase overall. There was faster increase in absorbance value for orange as compared to apple leading to faster formation of benzoquinone that is ripening of fruit as compared to apple.

\subsection{Protease}

The activity of protease was taken after every 2 minutes at $540 \mathrm{~nm}$. The change in absorbance value was noted after incubating the sample for 30 minutes to enhance the activity of Protease enzyme (Fig.4). Protease activity of tomato at 0 minute was found to be 1.55 . The mixture was then incubated for 30 minutes and the absorbance was noted after every 2 minutes. Absorbance was found to increase up to 6 minutes initially and then became constant.

In the extract of orange juice extract, the protease activity at 0 minute was found to be 1.38 . After incubating for 30 minutes the absorbance was noted after every 2 minutes. It was found to decrease for up to 6 minutes initially and then became constant.

\section{CONCLUSION}

The present investigation was focused on the enzymatic profile of Catechol oxidase and Protease in fruit/vegetable ripening. Catechol oxidase released from broken cells catalyzes the reaction to form benzoquinone i.e.Orange coloured complex and later form melanin. An increase in absorbance due to formation of benzoquinone in tomato, orange and apple juices proved the formation of benzoquinone i.e. increased activity of the enzyme implying to more ripening of fruits and vegetables as compared to carrot juice where the absorbance value was found to decrease implying less activity of enzyme. Protease enzyme (Fig.4) present in the tomato juice was not able to digest the egg albumin (substrate) leading to increase in absorbance, hence showing decreased activity whereas in orange juice the absorbance was found to decrease showing increased enzyme activity. This implies that protease plays an important role in ripening and softening of the fruit as compared to vegetable.

\section{Acknowledgements}

Authors are highly thankful to the Principal, Dr. P. Hemalatha Reddy and Dr. N.V.S.R.K. Prasad, Department of Botany, Sri Venkateswara College for availing the lab facilities.

\section{REFERENCES}

[1]. http://www.food-info.net/uk/colour/enzymaticbrowning.htm

[2]. http://www.saps.org.uk/.

[3]. Randall, D., Burggren, W. and French, K.1997. Eckert Animal Physiology: Mechanisms and Adaptations. Freeman, W. H. (ed.). Freeman and Company. New York. pp. 650-653.

[4]. Smith, L. S. 1989. Digestive functions in teleost fishes. In: Fish Nutrition. Halver, J. E. (ed.). London, pp. $31-421$.

[5]. Munilla-Morán, R. and Saborido-Ray, F.1996. Digestive enzymes in marine species. I. Proteinase activities in gut from redfish (Sebastesmentella), sea bream (Sparusaurata) and turbot (Scophthalmusmaximus). Comparative Biochemistry and Physiology 113B: 395-402.

[6]. Solomon, E. P., Berg, L. R., Martin, D. W. and Villee, C. 1996. In: Biology. (ed.). Saunders College Publishing, Philadelphia, pp. 982-993.

[7]. Alison Kingston-Smith, In Donnison, Paul Robson and Mike Theodorou. http://www.aber.ac.uk/en/media/99ch7.pdf.

[8]. Hedstrom, L. 2002. Serine protease mechanism and specificity. Chemical Reviews 102: 4501-4524.

[9]. Rawlings, N. D., Morton, F. R. and Barrett, A. J. 2006. MEROPS: The peptidase database. Nucleic Acids Research 34: $270-272$.

[10]. Otlewski J, Jelen F, Zakrzewska M, Oleksy A (2005). The many faces of protease-proteininhibitor interaction. EMBO J 24: 1303-1310

[11]. Page, M. J. and Di Cera, E. 2006. Role of Na and K in enzyme function.Physiological Reviews 86: 1049-1092.

[12]. http://www.enzymeessentials.com/HTML/protease.html.

[13]. http://thedomainfo.com/nutrition/nutrition facts/.

[14]. http://guide2herbalremedies.com/health-benefits-carrot-juice/. 